\title{
Fluoride mouthrinses provide clear reduction in caries in children
}

\author{
Are fluoride mouthrinses effective in preventing caries?
}

\begin{abstract}
Marinho VCC, Higgins JPT, Logan S, Sheiham A. Fluoride mouthrinses for preventing dental caries in children and adolescents (Cochrane Review). In Cochrane Library 2003; Issue 3. Oxford: Update Software

Data sources Sources of studies were the Cochrane Oral Health Group's Trials Register, Cochrane Central Register of Controlled Trials, Medline, Embase, SCISEARCH, SSCISEARCH, ISTP, BIOSIS, CINAHL, ERIC, Dissertation Abstracts and LILACS. Searches were also made by hand of journals and reference lists of articles, and contact was made with selected authors and manufacturers.

Study selection Studies included were randomised or quasi-randomised controlled trials with blind outcome assessment that compared fluoride mouthrinse with placebo or no treatment at least 1 year in children aged up to 16 years over. The main outcome was caries increment measured by the change in decayed, missing and filled tooth surfaces [D(M)FS].

Data extraction and synthesis The primary measure of effect was the prevented fraction (PF; ie, the difference in mean caries increments between the treatment and control groups expressed as a proportion of the mean increment in the control group). Meta-analyses were performed where data could be pooled. Potential sources of heterogeneity were examined by meta-regression.

Results Thirty-six studies were included, with 34 contributing data for meta-analysis (involving 14600 children). The $\mathrm{D}(\mathrm{M}) \mathrm{FS}$ pooled $\mathrm{PF}$ was $26 \%$ (95\% confidence interval, $23-30 \% ; P<0.0001)$. Heterogeneity was not substantial but was confirmed statistically $(P=0.008)$. No significant association between estimates of $\mathrm{D}(\mathrm{M}) \mathrm{FS} \mathrm{PF}$ and baseline caries severity, background exposure to fluorides, rinsing frequency and fluoride concentration was found. There is little information concerning possible adverse effects or acceptability of treatment in the trials included (Table 1).
\end{abstract}

Table 1. NNT at different caries increment levels to avoid one D(M)FS.

\begin{tabular}{lc}
\hline Caries increment $[\mathrm{D}(\mathrm{M}) \mathrm{FS} /$ year $]$ & NNT to avoid one $\mathrm{D}(\mathrm{M}) \mathrm{FS}$ \\
\hline 0.25 & $16.0(95 \% \mathrm{Cl}, 14.0-18.0)$ \\
2.14 & $1.8(95 \% \mathrm{Cl}, 1.6-2.0)$ \\
\hline
\end{tabular}

$\mathrm{D}(\mathrm{M}) \mathrm{FS}$, decayed, missing and filled tooth surfaces; NNT, number needed to treat; $\mathrm{Cl}$, confidence interval.

Conclusions This review shows that the supervised regular use of fluoride mouthrinse at two main strengths and rinsing frequencies is associated with a reduction in caries increment in children. There is a need for complete reporting of side-effects and acceptability data in fluoride mouthrinse trials.

\section{Commentary}

At the outset I congratulate the authors on an excellent review carried out according to Cochrane methods in an important area in preventive dentistry. Their objectives were to evaluate randomised or quasi-randomised controlled trials in children and adolescents, with blind assessment, looking at:

- efficacy and safety in preventing dental caries,

- influence of caries severity on the efficacy,

- influence of background exposure to fluoride on the efficacy, and

- influence of fluoride concentration or application on the efficacy.

To do this the authors examined 11 databases, augmenting this with searches of journals and article references by hand, plus contact with selected authors and manufacturers. Details of the number of articles they identified are difficult to comprehend (tabulating these would have helped) but my understanding is that more than 3000 were found, 292 were potentially eligible, 92 were assessed, and 36 were finally included which involved 14600 children. This shows the tremendous amount of work by the authors, and also how such strict inclusion criteria exclude much published work that might otherwise have been used in a narrative review. To me the temporal spread of the trials, 29 of which were double-blinded, is interesting: 10 from the 1960s, 19 from the 1970s, six from the 1980s and only one from the 1990s. This indicates how research interest has shifted and, in my opinion, reflects similar activity in dental caries epidemiology. The authors understandably remark on the variable quality of the trials: each study represents the state-of-the-art at the time it was made, showing how clinical trial methodology has advanced to the present.

The meta-analyses in the review clearly demonstrates that using fluoride rinses reduces caries rate, and that the baseline caries-severity influences the effect ie, the higher the caries severity the fewer are the children who require treatment to avoid one D(M)FS. Also, background fluoride use has little influence on the effect of fluoride mouthrinses in preventing caries. Unfortunately, lack of information on adverse effects or acceptability stifled that part of the review.

The ultimate question that anyone reading the review must ask is whether mouthrinses are applicable in local circumstances. For South Africa, my opinion is no, since cost-benefit considerations still favour water fluoridation. What is clear is that without this review it would have been much harder to come to this conclusion.

\section{Practice points}

- Fluoride rinses reduce caries.

- Fluoride rinses are more effective in high caries areas.

- Background fluoride use has little influence on the preventive effect of fluoride rinses.

Peter Cleaton-Jones

University of the Witwatersrand Dental Research Institute, Johannesburg, South Africa

Evidence-Based Dentistry (2003) 4, 85.

doi:10.1038/sj.ebd.6400221
Address for correspondence: Emma Tavender, Review Group Co-ordinator, Cochrane Oral Health Group, University Dental Hospital of Manchester, Higher Cambridge Street, Manchester M15 6FH, UK. E-mail: emma.tavender@man.ac.uk. 